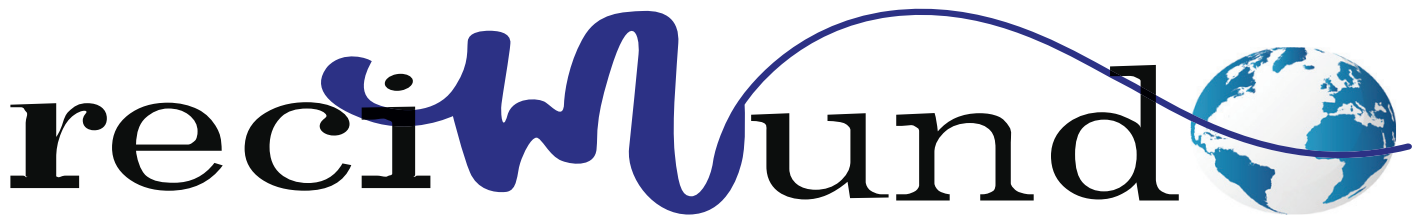

Revista Científica Mundo de la Investigación y el Conocimiento

DOI: 10.26820/recimundo/4.(1).enero.2020.13-25

URL: http://recimundo.com/index.php/es/article/view/710

EDITORIAL: Saberes del Conocimiento

REVISTA: RECIMUNDO

ISSN: 2588-073X

TIPO DE INVESTIGACIÓN: Artículo de Revisión

CÓDIGO UNESCO: 3213.15 Traumatología

PAGINAS: $13-25$

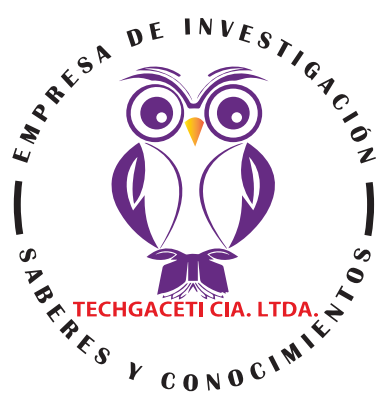

\title{
Utilidad del lavado peritoneal en el trauma abdominal
}

\section{Usefulness of peritoneal lavage in abdominal trauma Utilidade da lavagem peritoneal em trauma abdominal}

Mayra Estefanía Pazmiño Vargas'; Sabrina Esther Saltos Morán²; Javier Alejandro Coloma Pereira3; Matthew Leonardo Molina Villacres ${ }^{4}$

RECIBIDO: 18/12/2018 ACEPTADO: 29/03/2019 PUBLICADO: 01/12/2019

1. Médica; Investigadora Independiente; Guayaquil, Ecuador; mayrapazmino24@hotmail.com; (DD https://orcid. org/0000-0001-8236-3363

2. Médico; Investigadora Independiente; Guayaquil, Ecuador; sabrinasaltosm@hotmail.com; (D) https://orcid. org/0000-0002-8134-4328

3. Médico; Investigadora Independiente; Guayaquil, Ecuador; Javiercp589@hotmail.com; (D) https://orcid. org/0000-0001-7745-4666

4. Médico; Investigadora Independiente; Guayaquil, Ecuador; Mattmolinavillacres@gmail.com; (D) https://orcid. org/0000-0002-2665-098X

CORRESPONDENCIA

María Auxiliadora Cedeño Cevallos

auxicede1989@hotmail.com

Guayaqui, Ecuador

๑ RECIMUNDO; Editorial Saberes del Conocimiento, 2020 


\title{
RESUMEN
}

La evaluación de pacientes que presentan traumatismo abdominal, casos estables pero con la incógnita de si están comprometidos órganos internos. Generalmente estos casos se generan junto con traumas múltiples, y muchas veces en estado inconsciente, o en estado de shock. De tal forma, que se requiere de la aplicación de métodos acertados para el diagnostico preciso y eficientes, para proceder con la decisión quirúrgica requerida de forma rápida. De allí que los métodos auxiliares como el lavado peritoneal diagnostico. El presente estudio de investigación documental, analiza las bondades de este tipo de diagnostico en los diferentes tipos de traumas abdominales que se presentan en salas de urgencias y que requiere de toma de decisiones acertadas y oportunas para su intervención quirúrgica y / o tratamiento requerido. Se analizará detenidamente, las disyuntiva sobre si efectivamente el Lavado peritoneal diagnostico, es de utilidad en los traumas de abdomen de tipo penetrante abierto, con el propósito de instar a continuar las investigaciones científicas que generen aportes teóricos practico sobre este método de gran utilidad ante el trauma abdominal.

Palabras clave: Lavado Peritoneal, Trauma Abdominal, Cavidad Peritoneal, Diagnósticos, Traumas, Procedimientos Quirúrgicos.

\begin{abstract}
The evaluation of patients presenting with abdominal trauma, stable cases but with the uncertainty of whether internal organs are involved. Generally these cases are generated along with multiple traumas, and often in an unconscious state, or in a state of shock. Thus, it is required the application of accurate and efficient diagnosis methods to proceed with the required surgical decision quickly. Hence, auxiliary methods such as diagnostic peritoneal lavage. The present documentary research study analyzes the benefits of this type of diagnosis in the different types of abdominal traumas that occur in emergency rooms and that requires accurate and timely decision making for their surgical intervention and / or required treatment. It will be analyzed in detail, the dilemma about whether indeed the diagnostic peritoneal lavage, is useful in traumas of open penetrating abdomen, with the purpose of urging to continue the scientific investigations that generate practical theoretical contributions on this method of great utility before the abdominal trauma.
\end{abstract}

Keywords: Peritoneal Wash, Abdominal Trauma, Peritoneal Cavity, Diagnoses, Traumas, Surgical Procedures.

\section{RESUMO}

A avaliação de pacientes com trauma abdominal, casos estáveis, mas com a incerteza quanto à presença de órgãos internos. Geralmente, esses casos são gerados juntamente com vários traumas, e geralmente em estado inconsciente ou em estado de choque. Portanto, é necessária a aplicação de métodos de diagnóstico precisos e eficientes para prosseguir rapidamente com a decisão cirúrgica necessária. Portanto, métodos auxiliares, como lavagem peritoneal de diagnóstico. O presente estudo documental analisa os benefícios desse tipo de diagnóstico nos diferentes tipos de traumas abdominais que ocorrem em pronto-socorros e que requerem tomadas de decisão precisas e oportunas para sua intervenção cirúrgica e / ou tratamento necessário. Será analisado em detalhes, o dilema sobre se de fato a lavagem peritoneal diagnóstica é útil em traumas de abdômen penetrante aberto, com o objetivo de insistir em continuar as investigações científicas que geram contribuições teóricas práticas sobre esse método de grande utilidade antes da cirurgia abdominal. trauma.

Palavras-chave: Lavagem Peritoneal, Trauma Abdominal, Cavidade Peritoneal, Diagnósticos, Traumas, Procedimentos Cirúrgicos. 


\section{Introducción}

Una de las principales complicaciones a los que se enfrenta el cirujanoen Servicios de Urgencia, es la evaluación de pacientes que presentan traumatismo abdominal, casos estables pero con la incógnita de si están comprometidos órganos internos. Generalmenteestos casos se generan junto con traumas múltiples, y muchas vecesen estado inconsciente, o en estado de shock. "La lesión inadvertida del contenidoabdominal sigue siendo una causa desafortunadamentefrecuente de muerte evitable y producto deldiagnóstico omitido o retrasado de las lesiones abdominales" (Espinal, Ventura, \& Garcia, 1995); de tal forma, que se requiere de la aplicación de métodos acertados para el diagnostico preciso y eficientes, para proceder con la decisión quirúrgica requerida de forma rápida. De allí que los métodos auxiliares como el Lavado Peritoneal Diagnostico (LPD), la Paracentesis, ultrasonido grafía y tomografía axial computarizada, sirvan para el logro de este objetivo. En el presente estudios investigativo se presta especial atención al Lavado Peritoneal (LPD) como principal método para tratar y diagnosticar el trauma abdominal, para analizar sus bondades y los planteamientos de especialistas en cuanto a su aplicación.

Las videotecnologías con el pasar de los años han cobrado mayor fuerza en los servicios de urgencias, como desarrollo vertiginoso que en menos de una década de la "colecistectomía", ha tomado protagonismo principalmente "Laparoscópica" marcando un cambio drástico en la práctica de la cirugía general y en los servicios de emergencias específicamente. Laparotomía exploradora es un método terapéutico para el trauma abdominal y sobretodo si hay sospecha de lesiones viscerales, reparando de forma inmediata las lesiones graves.

En tal sentido, aquellos pacientes que presentan inestabilidad hemodinámica debe valorarse sus condiciones con exámenes que ofrezcan resultados rápidos de manera que pueda tomarse una decisión inmediata sobre la pertinencia quirúrgica y establecer si efectivamente debe aplicarse laparotomía, para lo cual la paracentesis, el lavado peritoneal diagnóstico y la ultrasonografías son los principales métodos que permiten diagnosticar su pertinencia. Cabe resaltar, que este proceso genera disminución en la incidencia de morbi-mortalidad.

"Hay un gran afán de readiestramiento de los cirujanos y reequipamiento de las salas de operaciones. Con esta transformación permanente de videotecnología, el cirujano y personal entrenado está avanzando con rapidez por ser un proceso dinámico en evolución y revisión constante para alcanzar a ser un método seguro, benéfico y efectivo en manos bien entrenadas." (Espinal , Ventura, \& Garcia, 1995)

Aún cuando la incidencia de laparotomías innecesarias por trauma abdominal es relativamente alta, sigue siendo el principal procedimiento quirúrgico, por lo que el lavado peritoneal cobra mayor significancia; en este mismo sentido dicha incidencia variará de acuerdo al mecanismo de lesión y el método auxiliar de valoración-diagnóstico preoperatorio que sea utilizado. Por tal motivo, en el presente estudio se ahondará en las bondades del lavado peritoneal que proporcione un diagnóstico adecuado y una pertinente intervención quirúrgica.

\section{Método}

La metodología utilizada en el presente estudio es la investigación documental a través de la revisión sistemática de bibliografía, análisis e interpretación de literatura científico académica disponible, refiriendo a diccionarios médicos, identificación de conceptos básicos en torno al tema planeado, revisión de estudios aplicados y constatación con la bibliografía analizada.

Se seleccionó la información de acuerdo a los criterios de pertinencia y correlatividad temática, en publicaciones, revistas, artí-

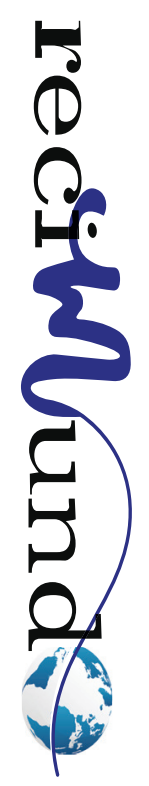


culos académicos y científicos, originales, revisiones sistemáticas, protocolos, libros, boletines, folletos, tesis de posgrado y doctorado, noticias científicas, entre otros documentos e información de interés científico y académico.

En efecto, un cúmulo de aproximadamente 60 contenidos bibliográficos, permite consolidar un análisis descriptivo sobre los traumas abdominales, los diferentes tipos y causas; así como la formas de diagnostico existentes, con detenimiento y profundización en el lavado peritoneal y su funcionalidad para el tratamiento quirúrgico apto y proporciona conocimiento en torno a la vanguardia tecnológica- científica de hoy.

Se efectúa el análisis, interpretación y discusión de dicho material tratado como evidencia científico académica, y se determinó el criterio grupal de las ideas y planteamientos expuestos.

\section{Resultados}

Trauma abdominal:Se habla de trauma abdominal cuando esta cavidad del cuerpo orgánico es lesionada por acción violenta de agentes externos, en la pared abdominal y/o en su interior. El trauma puede ser incluso causado como parte de politraumatismos y su magnitud varia.

En tal sentido, el trauma abdominal puede producirse de forma directa o indirectamente. En la primera el agente impactará el abdomen, mientras que se refiere a traumas indirectos cuando son resultado de un contragolpe, tales como caídas y por híper presión intrabdominal.

\section{Trauma contuso o cerrado}

El trauma cerrado "se produce como consecuencia de una combinación de fuerzas de compresión, deformación, estiramiento y corte. La magnitud de estas fuerzas está en relación directa con la masa de los objetos involucrados, su aceleración y desaceleración y su dirección relativa durante el impacto. El daño ocurre cuando la suma de estas fuerzas excede las fuerzas cohesivas de los tejidos y órganos involucrados. (Presswalla, 1978)

El impacto directo y las fuerzas compresivas son probablemente las causas más comunes de traumas significativos y la severidad se puede estimar si uno conoce la fuerza y dirección del impacto, al igual que el tamaño del área de contacto en el paciente(Presswalla, 1978).

Los órganos más vulnerables del cuerpo humano son los que contienen gas, como los pulmones y el intestino. En el trauma contuso en general, los órganos más lesionados son el hígado, bazo, mesenterio y riñón. (Comité de Trauma de Colegio Americano de Cirujanos, 2004)

Según el analisis realizado por (Pacheco, 2011) se puede mencionar del trauma cerrado o contuso:

- Que se produce con alta incidencia.

- El examen físico es poco confiable.

- Solo un 6\% aproximadamente de los pacientes con traumas abdominales cerrados requerirán laparotomía.

Los diagnósticos en traumas cerrados, que se pueden realizar son mediante el Lavado peritoneal diagnóstico, Ultrasonido y Tomografía Axial.

La aplicación de estos métodos dependerá del estado del paciente, así por ejemplo si el paciente con trauma abdominal ingresa estable, o luego del manejo inicial en salas de urgencia es estabilizado, se podrá proceder a aplicar diagnósticos para determinar si existe o no indicación para realizar una intervenciónquirúrgica.

Según estudio realizado por(Espinal, Ventura, \& Garcia, 1995); "se estima que solamente 5\%-10\% de los pacientescon trauma cerrado y que alrededor de 35\% de los pacientes heridos porarma corto punzante, requieren de una intervención quirúrgica.". 
Por su parte, existen casos de pacientes a los cuales la indicación es efectuar el procedimiento laparotomía, y realizar la intervención quirúrgica indiferentemente del resultado del manejo inicial. Por ejemplo pacientes que presentan "Inestabilidad hemodinámica" la cual muestra hipotensión y no responde a la administración de solución electrolítica balanceada, en estos casos, usualmente implica la presencia de una hemorragia intra abdominal no controlada, ante la cual es completamente inapropiado persistir en la administración de líquidos endovenosos, tratando de normalizar los signos vitales; se debe en cambio, para proceder a realizar la hemostasia, someter al paciente de inmediato a una laparotomía. De igual forma, se aplicará si el paciente muestra signos de irritación peritoneal. Evisceración (excepto del epiplón) y Hematemesis. En cuanto a las heridas trans-abdominal por arma de fuego, se debe proceder a identificar a quienes requieren cirugía, para realizar así un manejo selectivo, que significa seleccionar o identificar a aquellos pacientes en los cuales la intervención quirúrgica es necesaria. En realidad, realizar laparotomías innecesarias puede ser tan nocivo como no realizarlas en los que las requieren, como lo demuestran las cifras de morbilidad e inclusive mortalidad por laparotomías no terapéuticas.

En el efecto, se procede a una revisión sobre los métodos de diagnósticos como el lavado peritoneal y sus beneficios en este sentido.

\section{Lavado peritoneal}

El Lavado Peritoneal Diagnóstico ( LPD) es un método diagnóstico rápido, sencillo tanto en su realización e interpretación, es seguro y sensible en el diagnóstico de las lesiones inrraabdominalesen el paciente politraumatizado, se estima que el 20 a50\% de las muertes en el trauma cerrado pueden prevenirse sise realiza rápidamente su diagnóstico y tratamiento

Desde la descripción inicial por Reo en 1965 en 28 pacientes traumatizados, el LPD ha sido estudiado,extensivamente y aún cuando existan hoy en día métodos más complejos como la arteriografía, la ecosonografía y la tomografía computarizada, su uso no ha perdido importancia, ya que es de gran utilidad en el diagnóstico de lesiones en el trauma abdominal cerrado. También juega un rol importante en el trauma abdominal penetrante ocasidonado por arma blanca o de fuego en los pacientes hemodinámicamente estables donde la penetración a la cavidad peritoneal, o el compromiso de los órganos intra-abdon1inales es dudoso, tal es el caso de heridas que comprometen el abdomen o el tórax con trayectos tangenciales o superficiales en la región dorsal, en los flancos, en la pelvis, en el tórax inferior y en la región glútea.

Sin embargo ante traumas penetrantes su utilización continúa siendo debatida por algunos autores, ya que no permite detectar lesiones de diafragma y retroperitoneales en algunos casos de estudio, aunado a ser un método invasivo, con baja especificidad.

El Lavado peritoneal diagnósticoes un procedimiento rápido y preciso que diagnostica lesiones intrabdominales en pacientes con trauma contuso, este procedimiento sustituyó a la punción abdominal, su uso ayuda a realizar la laparotomía más pronto, más expedita, con menos pérdida de sangre, evitando las transfusiones y su utilidad se precisa entre otros casos, cuando el paciente no responde adecuadamente a la resucitación y el ultrasonido es negativo,

\section{Técnica:}

Debe ser practicado por el cirujano. Se puede realizar con técnica abierta, semiabierta o cerrada Es concocida la técnica abierta como método seguro y rápido, sin embargo el método cerrado con la técnica de Seldinger, es adecuado cuando se realiza por personal familiarizado con la mima.

\section{Se debe evacuar vejiga y estómago.}

2. Preparar la piel con soluciones antisép-

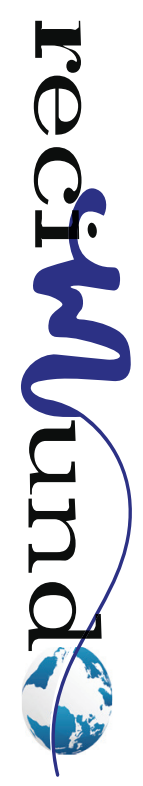


ticas.

3. Infiltrar con anestésico local con epinefrina la línea media a $1 / 3$ de distancia entre el ombligo y la sínfisis púbica.

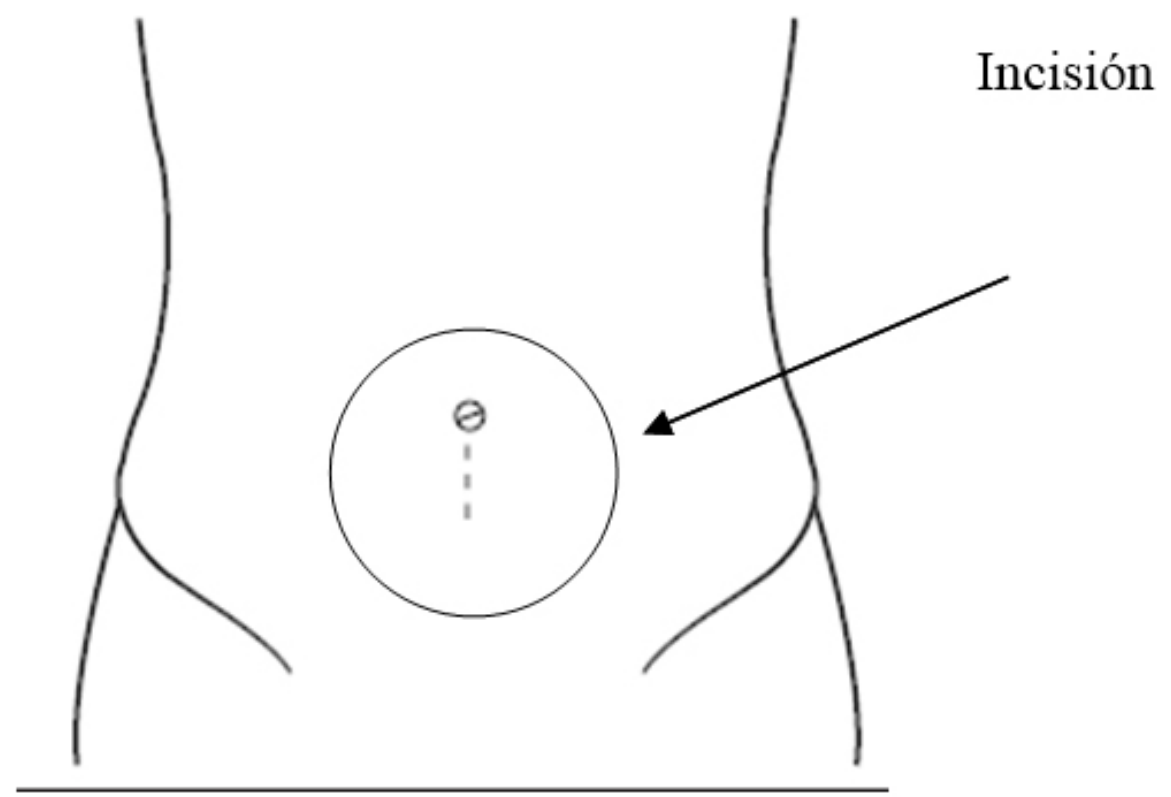

Figura 1. Incisión para el Lavado Peritoneal Diagnóstico

Fuente: (Ferrada, Garcia, Cantillo, Aristizábal, \& Abella)

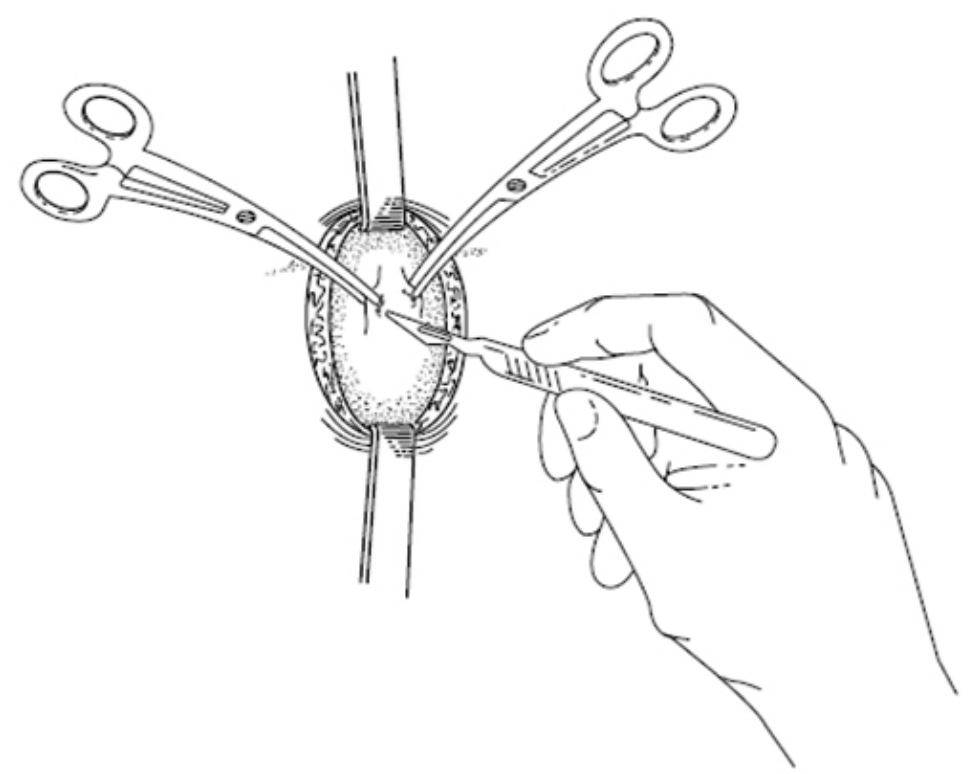

Figura 2. Lavado Peritoneal Diagnóstico

Fuente: (Ferrada, Garcia, Cantillo, Aristizábal, \& Abella)

Se introduce un catéter en la cavidad peritoneal para aspirar sangre o líquidos. Si no se extrae nada, se infunde un litro de solución salina normal tibia (o $10 \mathrm{ml} / \mathrm{Kg}$ en los niños). Después de asegurar que los líquidos fue-
4. Incidir verticalmente la piel y tejidos subcutáneos hasta la aponeurosis 


\section{Se considera positivo:}

- Extracción de $10 \mathrm{ml}$ de sangre roja al aspirar

- >100.000 glóbulos rojos /cc

- >500 glóbulos blancos /cc

- Presencia de bacterias

- Presencia de bilis

- Presencia de partículas de comida

\section{Ventajas del lavado peritoneal}

- Introducción a la cavidad peritoneal de una solución lactato de ringer y su recuperación posterior con fines diagnósticos

- Se introduce solución salina a través de una incisión en el abdomen

- Bajo costo

- Mínima morbilidad

- Facilita la toma de decisiones

- Fácil ejecución

- Alta tazas de precisión y certeza en diagnósticos

\section{Lavado Peritoneal Diagnóstico en Trauma contuso o cerrado}

- Incidencia de complicaciones es menor si se realiza contécnica abierta

- Los falsos positivos aumentan en presencia de Fx pélvica

- Superior al TAC en detección de lesión mesentérica

- No descarta confiablemente lesiones retroperitoneales

- A pesar de los estudios alternos en trauma abdominal en recientes estudios autores recomiendan el LPD cuando se sospecha lesión de víscera hueca y los hallazgos del US, la T.C. y el examen físico no son concluyentes
- En el paciente inestable con US y T.C indeterminados el LPD permanece como lamodalidad diagnóstica de elección.

\section{Trauma abdominal penetrante: Positivo}

- Hematiesmayor que $100.000 \mathrm{~mm}$

- Hematies menor que 50.000 mm

- Leococitos $100 \mathrm{~mm}$

- Amilasa menor que 75 U1/L

- Indeterminado (trauma abd cerrado)

- Hematies mayor que 50000 y menor que $100, .000 \mathrm{~mm}$

- Leucocitos mayor que 100 y menor que $500 \mathrm{~mm}$

- Amilasa mayor que 75 y menor que 175 I/L

\section{Complicaciones}

- Sangrado en la incisión o punción

- Preformación intestinal por el catéter y peritonitis secundaria

- Laceración de la vejiga

- Lesión de vasos mayores o de otros órganos abdominales.

\section{EI Lavado Peritoneal Diagnóstico}

(Feliciano, 1995) plante que al hacer un análisis de diversas modalidades de diagnóstico en el traumatismo abdominal (lavado peritoneal, ultrasonografía, tomografía computarizada y arteríografía) concluye en que el lavado peritoneal diagnóstico tiene una función primaria en la comprobación de la hemorragia o la contaminación intrabdominal en pacientes con traumatismo cerrado, o en algunos pacientes estables penetrantes, sin embargo, pasa por alto lesiones pequeñas y grandes del diafragma y no ayuda a descartar la existencia de lesiones de los tejidos u órganos retroperitoneales.

Generalmente luego de mecanismo pene-

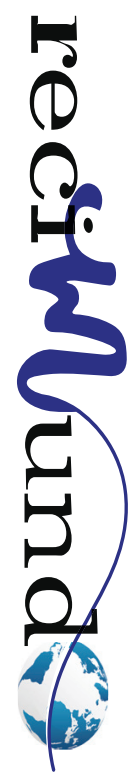


trante, la toma de decisión es más fácil. No obstante, entre especialistas existen controversias en torno al uso adecuado el Lavado Peritoneal, por ejemplo de (Oreskovich \& Carrico, 1983), quienes basado en el hecho de realizar una laparotomía exploradora sistemáticamente después de cada herida penetrante de abdomen por arma blanca plantearon que conlleva una tasa alta delaparotomía negativa (31-63\%) y de que hay una pequeña pero significativa mortalidad, consecuencia de anestesia en este grupo de pacientes con laparotomía no productivas y propusieron un algoritmo para el manejo selectivo de las heridas abdominales por arma blanca empleando el examen físico, la exploración local de la herida y lavado peritoneal diagnóstico.

(Huizinga, Baker, \& Mtshali, 1987) propone un manejo selectivo aún en presencia de demostración evidente de penetración peritoneal, tal como la salida de epiplon siempre y cuando no existan indicaciones francas de la laparotomía tales como signos peritoneales o shock sobre esta última opinión, los autores comparten más el criterio de (Burnwit \& Thal, 1986), de que la salida de epiplón a través de una herida por arma blanca es todavía una indicación para laparotomía.

La decisión de realizar una laparotomía por traumatismo abdominal cerrado resulta mucho más difícil y compleja que el traumatismo abierto, porque la lesión estructural es menos manifiesta y frecuentemente enmascarada por lesiones, no debiendo fiarse únicamente del examen físico inicial, lo que obliga a que aparte de los estudios del laboratorio y radiografías, llevar a cabo en estos paciente el lavado peritoneal diagnóstico que constituye un medio rápido, poco costoso, preciso y relativamente seguro de modalidad diagnóstica para valorar pacientes con traumatismo abdominal cerrado, lo que desde esa fecha ha sido repetidamente confirmado en la literatura. En una revisión colectiva de 32 series, involucrando a 10,358 pacientes con trauma abdominal cerrado, la especificidad del lavado peritoneal diagnóstico fue de $97.3 \%$, la tasa de falsos positivos y falsos negativos fue de $1.4 \% \mathrm{y}$ de $1.3 \%$ respectivamente.

La tasa de complicaciones al practicarse el lavado peritoneal diagnóstico es en general menor del $1 \%$.

Gráfica 1. Trauma Abdominal Cerrado Paciente Estable.

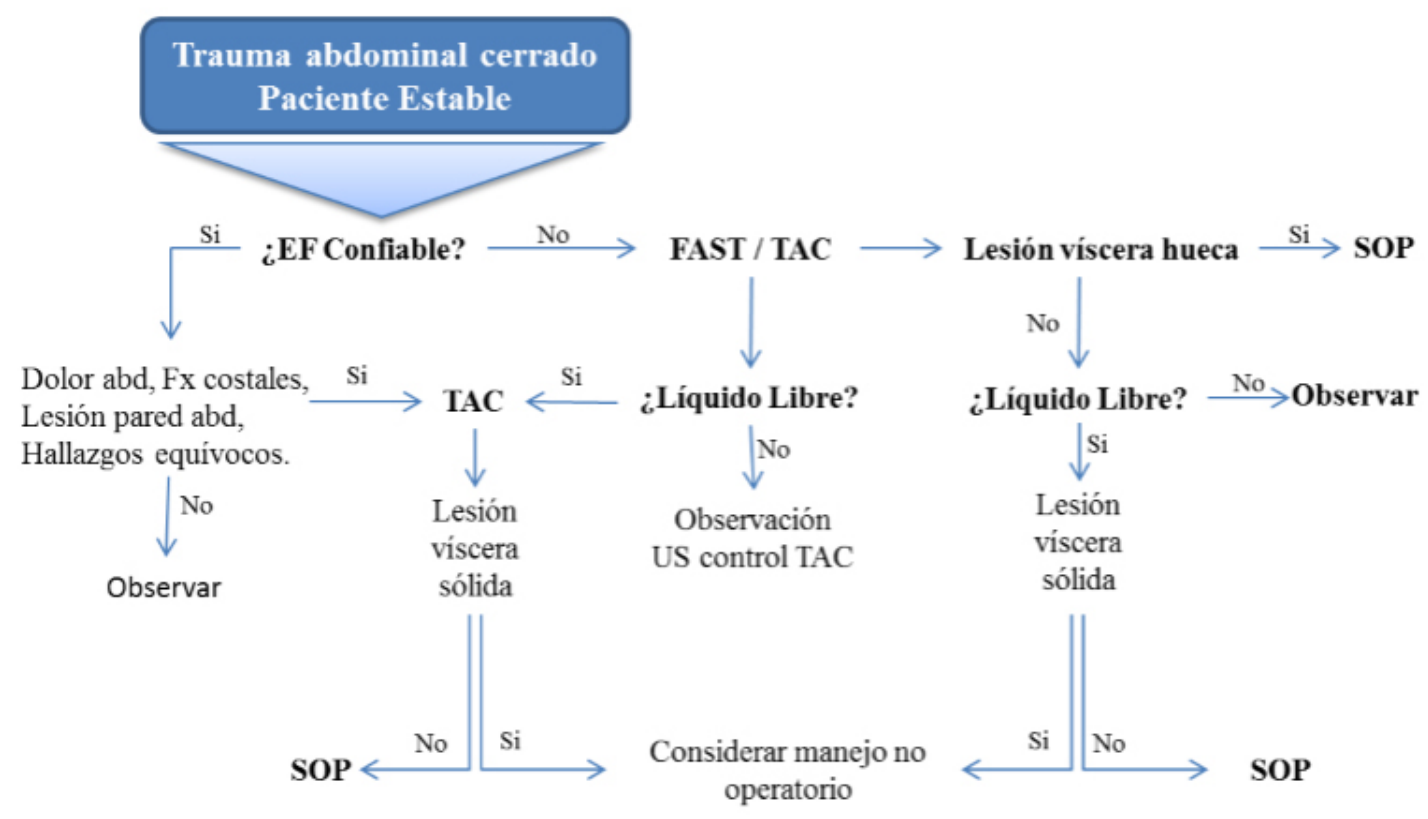

Fuente: (Saldarriaga L.) 
Gráfica 2. Trauma Abdominal Cerrado Paciente Inestable.

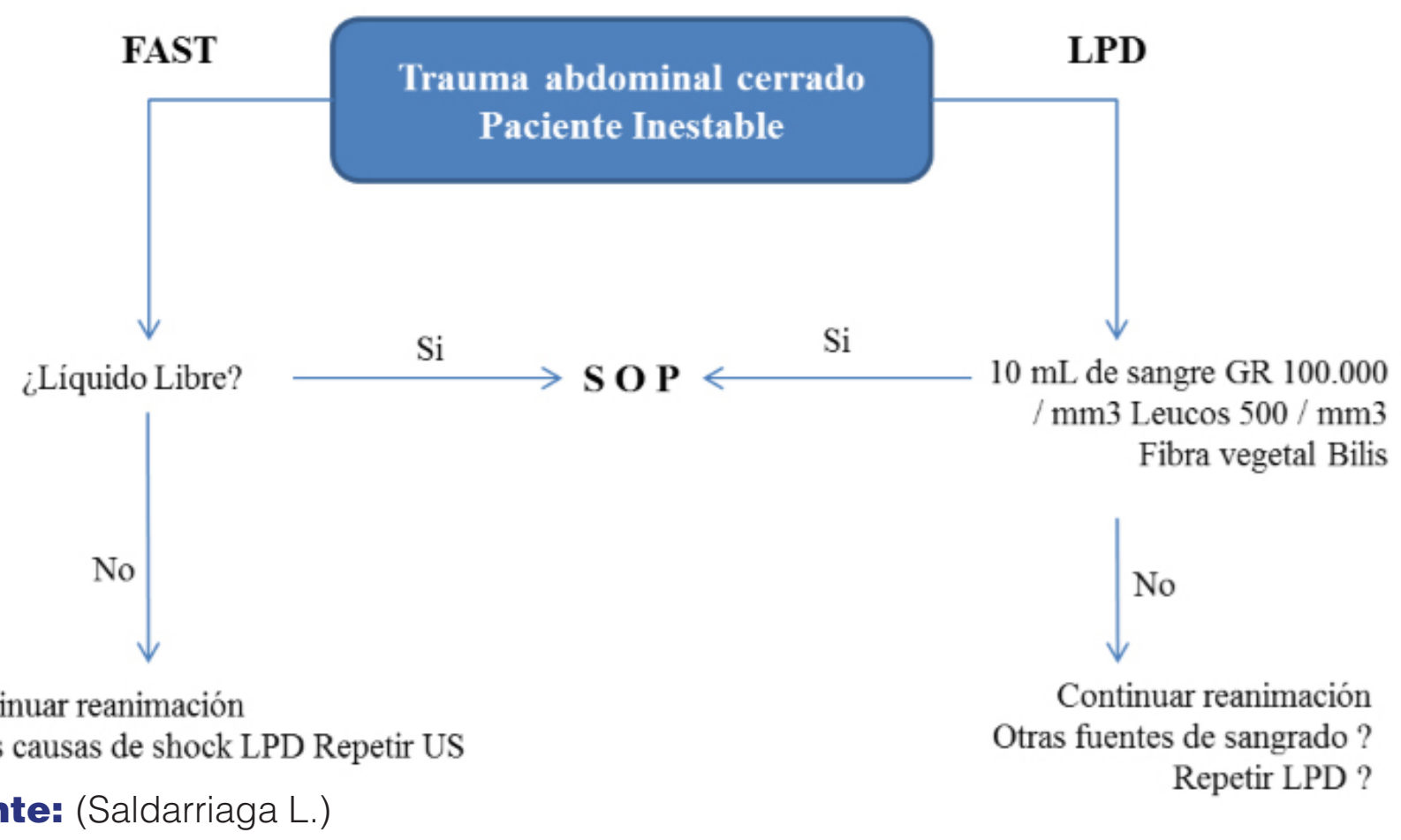

\section{Traumatismo abierto}

Penetrante o no penetrante

\section{Trauma penetrante}

Las heridas por arma blanca y las por arma de fuego de baja velocidad causan daño al tejido por laceración o corte. Las heridas por proyectiles de alta velocidad transfieren mayor energía cinética a las vísceras abdominales, teniendo un efecto adicional de cavitación temporal, y además causan lesiones abdominales en su desviación y fragmentación.

En relación a los proyectiles disparados por un arma de fuego, el daño no solo depende de la energía cinética (masa $x$ velocidad), sino también depende de la estabilidad del proyectil (dada por las estrías del cañón). Es importante diferenciar las heridas penetrantes producidas por arma de fuego, que tienen una trayectoria muchas veces impredecible, de aquellas producidas por objetos punzantes, ya que la trayectoria es más directa y la relación anatómica entre el área de penetración y las lesiones de órganos internos es más cercana. La posibilidad de requerir cirugía, de complicarse y morir, es mayor en las heridas por arma de fuego que por arma blanca, y mayor por heridas múltiples que únicas. Los órganos que tienen mayor posibilidad de lesionarse son intestino delgado, hígado, estómago, colon y estructuras vasculares .

Es útil el enfoque topográfico, propuesto por González y García (Cali, Colombia), los que dividen el abdomen en áreas anatómicas, con diferente probabilidad de lesión de estructuras específicas, manifestaciones clínicas, alcance de las pruebas diagnósticas empleadas y abordaje. La probabilidad de lesiones se detalla a continuación:

- Abdomen anterior: todas las vísceras intrabdominales: yeyuno, íleon, colon, hígado, bazo, mesenterio y estómago.

- Toracoabdominal: diafragma, órganos torácicos y abdominales.

- Lumbar o abdominal posterior: riñones, uréteres, páncreas, duodeno, grandes vasos abdominales, cara posterior de colon ascendente y descendente. 
- Pélvica: recto, vejiga, uréteres distales, uretra, órganos genitales femeninos y masculinos, arterias y venas ilíacas y sus ramas (4).

\section{Utilidad del lavado peritoneal en el Trau- ma Abdominal}

El trauma abdominal cuenta con una alta morbilidad y mortalidad y el grupo etario mayormente afectado son los jóvenes que se encuentran en edad productiva.

Su manejo debe ser agresivo, de allí la importancia de un método eficaz para establecer si existen o no lesiones intrabdominales. La tardanza o el no diagnosticar una lesión importante intra-abdominal en este tipo de pacientes acarrea según se ha reportado en algunas series una mortalidad del $56 \%$.

Es importante resaltar que un gran grupo de pacientes eran operados en el pasado por la presunción de existir lesión intra-abdominal, no evidenciándose lesiones en la laparotomía. EI LPD es una extensión de la vieja técnica de aspiración con aguja de la cavidad perironeal, llamada paracentesis y que fue descrita por Saloman en. 1906, con el fin de diagnosticar peritonitis bacterianas. No es sino en 1946 cuando W right y col. aumentan la precisión de la paracentesis • al aspirar los cuatro cuadrantes del abdomen en pacientes traumatizados. La rata de exactitud de la paracentesis era del87.6\%, con un rango entre 69 y 93\%. Su problema estribaba en el alto porcentaje de falsos negativos los cuales alcanzaban el 9.4\% (16).

En 1965 Root y col. reportan la primera experiencia con el uso del LPD en 28 pacientes traumatizados, la Exactitud diagnóstica que alcanzó el 100\%, actualmente después de haber sido estudiado extensivamente se mantiene en un 97.3\%, estableciéndose la rata de falsos psicivos y negativos en I. 4 y 1.3 respectivamente. Su diferencia a la paracentesis consiste en que un LPD negativo provee una fuerte evidencia de no existir lesión intraperitoneal importante.
Desde su descubrimiento ha sido puesto a prueba por Innumerables métodos diagnósticos, los cuales muchas veces no son accesibles en la sala de emergencia, necesitando además una interpretación complicada. Tal es el caso de la tomografía computarizada ( TC) que no ofrece ninguna ventaja diagnóstica sobre el LPD y que tan solo pudiera ser un método alterno en aquellos casos donde no se pueda realizar el LPD. La diferencia en exactitud fue de $60 \%$ para el TC la cual aumentó a $85 \%$ al ser revisada nuevamente, contra un $97 \%$ para el LPD. Gruessner y coL ( 11 ), estudiaron la exactitud del LPb y. la del écosonograma abdominal demostrando estadísticamente la superioridad del LPD.

Las lesiones retroperitoneales que comprometen al páncreas, duodeno y colon no pueden ser diagnosticadas con el LPD. La decisión de realizar una laparotomía en este tipo de pacientes con un LPD negativo descansaría principalmente sobre la agudeza del examen clínico.

La utilización de métodos diagnósticos de extensión como los estudios radiológicos, la arteriografía, endoscopía y LPD son complementarias en el diagnóstico de las lesiones pancreato-duodenales, cabe resaltar que en muchos de estos pacientes existen concomitantemente lesiones intraperitoneales, a nivel de vísceras o grandes hematomas retroperitoneales que pudiesen tener apertura a la cavidad perítoneal.

La interpretaci6n de los resultados obtenidos del LPD han sufrido variaciones substanciales a través del tiempo. Para (Root, HAuser, Mckinley, Lafave, \& Mendiola, 1965), la positividad consistía en un líquido con coloración más oscura que el salmón rosado, consideraban además la amilasa, pigmentos biliares así como la presencia de bacterias. Posteriormente se estableció que un mililitro de sangre én un litro de solución salina arrojaba una cuenta de 5 a 7 mil células por milílitro, mientras que 100.000 células por mililitro equivalen a un he1na- 
tocrito del 1\%. Con respecto a los otros, la presencia de bilis indica lesión de asas intestinales, así como la presencia de bacterias gram negativa.

La utilidad del LPD en el trauma penetrante, tanto en las heridas por arma blanca como en las ocasionadas por arma de fuego es constatada a través del análisis de estudios realizados, que arrojaron exactitud sensibilidad y especificidad del $100 \%$ en el uso del LPD para este tipo de trauma.

"El manejo tradicional de los pacientes con heridas por arma blanca situadas a nivel del tórax inferior y en el abdomen ha sido la laparotomía exploradora, la controversia se presenta en los pacientes que ingresan hemodinámicamenre estables ya que actualmente se tiende a tomar una conducta conservadora, esto debido a la alta íncidencia de laparotomías negativas" (Isaac L, y otros, 1989).

En el estudio realizado por Thal muestra la tendencia en disminución de laparotomías negativas reportada por quien logró disminuir de $69.9 \%$ a $4.1 \%$ la laparotomías negativas al complementar la exploración de la herida con el LPD.

Por su parte, en estudio realizado en el Hospital de Vargas, Caracas 1989 , según narra el archivo del hospital, en un estudio, donde se manejaron 20 pácientes con heridas por arma blanca quienes ingresaron estables sin signos de peritonitis y donde la penetración era dudosa en la exploración de la herida En 5 pacientes el LPD fue positivo y huvo tan solo un fa1so positivo, no hubo falsos negativo y los resultados arrojaron una exactitud del $95 \%$, una sensibilidad del $100 \%$ y una especificidad del $94.4 \%$, y aunque no alcanza los valores observados en el trauma cerrado se encuentran en un rango aceptable para ese grupo de pacientes con heridas por arma blanca -haciendo hincapié de su estabilidad hemodinámica, de la ausencia de signos de irritación peritoneal y donde la exploración de la herida es dudosa.
(Isaac L, y otros, 1989)

100\% y una especificidad del 94.4 ó ,y pensamos que aunque no alcanza los valores observados en el traumacerrado se encuentran en un rango aceptable para ese grupo de pacientes con heridas por arma blanca ( 22 ) .haciendo hincapié de su estabilidad hemodinámica, de la ausencia de signos de irritación peritoneal y dondela exploración de la herida es dudosa.

En los 20 pacientes estudiados con heridas por arma de fuego y que se encontraban en las mismas condiciones que los anteriores los resultados fueron muy parecidos al grupo anterior aunque la especificidad disminuyó a un $91.6 \%$.

Moore y col. ( $13 \mathrm{~L}$ en un estudio de 245 pacientes claramente demostraron que el 96\% de los pacientes con penetración peritonea1 tenían lesiones intra-perironeales significativas. El LPD fue utilizado en 34 pacientes siendo positivo en 17 de ellos, en 14 se obtuvo más de $10 \mathrm{cc}$ de sangre a la aspiración inicial y en los 3 restantes se consrar6 más de 100.000 GR/ ce y en todos ellos había lesión intra abdominal significativa; 17 LPD fueron negativos o dudosos, en ellos dos desarrollaron signos de irritación peritoneal posteriormente, arrojando un porcentaje de falsos negativos del $6 \%$.

\section{Resultados de casos practicos revisa- dos:}

De acuerdo a los resultados se concluye que el LPD es un método diagnóstico senciIlo tanto en su realización como en su interpretación además de ser seguro y sensible en el diagnóstico de las lesiones intra-abdominales en los pacientes con trauma cerrado y penetrante. En nuestra emergencia donde no hay In posibilidad muchas veces de práctica $r$ exámenes sofisticados representa una alternativa viable pür sus características anteriormente descritas. Creemos que se deben establecer muy bien los parámetros para indicar a los pacientes con trauma penetrantes debido a que su exac- 
titud y su especificidad son menores a las observadas en el trauma cerrado abdominal'

\section{Conclusiones}

El Lavado Peritoneal Diagnostico (LPD), TAC, US y laparoscopía son técnicas muy sensibles a la presencia de líquido intraabdominal, pero sólo LPD y laparoscopía puede identificar el carácter del líquido (sangre o contenido del intestino delgado) y permite reducir el número de laparotomías innecesarias, de alli la importancia que reviste ante traumas abdominales.

Sin embargo, debe profundizarce en estuis que analicen la confiabilidad del metodo aplicado para lesiones de vísceras huecas, ya que ninguno de éstos tiene tal propiedad. La TAC y US pueden no detectar lesiones intestinales debido a que los volúmenes de líquido son menores a los límites de identificacion o pueden ser confundidos con pequeña cantidad de sangre. Ni LPD ni la US permiten localizar con precisión los órganos lesionados, en tanto que TAC y la laparoscopía si pueden detectarlos.

El LPD es de suma utilidad en pacientes con trauma abdominal contuso hemodinámicamente estables ya que está indicada la laparoscopía específicamente en aquellos de difícil diagnóstico de lesión visceral, con examen clínico y de laboratorio e imágenes no contributorio, cuando la observación puede ser peligrosa o la intervención quirúrgica inútil, puede tener alta posibilidad de morbimortalidad, de allí la pertinencia del Lavado, igualmente la utilidad en pacientes politraumatizados, en traumatismos encefálicos asociados a inconciencia y uso de alcohol o drogas.

Independientemente del mecanismo de lesión, los pacientes con trauma abdominal penetrante y signos vitales anormales o inestables y los que presentan peritonitis deben ser sometidos a laparotomía sin demora. Cuando se encuentran estables se realiza exploración local de la herida.
Si la pared abdominal anterior no ha sido penetrada el paciente es dado de alta. Si se encuentra penetrada, el LPD pondrían de manifiesto sangre o líquido en la cavidad peritoneal. Sin embargo el EI LPD no se considera la piedra angular del manejo de pacientes con trauma abdominal por arma de fuego.

La laparoscopía, con casi un siglo de edad, ha resurgido el interés de los cirujanos como una técnica para valorar a los pacientes con posible trauma abdominal, su aplicación se ve limitado por la posibilidad de otros procedimientos diagnósticos que pueden ser más adecuados para situaciones particulares y más precisos para ciertos tipos de lesiones, tal es el caso del LPD. Sus mejores aplicaciones podrían ser los casos estables con heridas penetrantes, que disminuyen la tasa de laparotomías innecesarias. Permite lograr fácilmente la hemostasia en lesiones menores de vísceras sólidas y también permite realizar reparaciones de daños mínimos de estómago, intestino y colon en casos seleccionados.

El entusiasmo excesivo por la laparoscopía en casos de traumatismo puede dar por resultado un abuso, cuando son más apropiadas otras medidas diagnósticas o la simple observación, puesto que incrementa los costos y los riesgos de la asistencia sin mejorar los resultados; sin embargo, así como va evolucionando puede ser un auxiliar muy valioso para el cirujano y constituye una gran promesa que requiere sin duda investigación a fondo en cuanto a su bondad diagnóstica y sus aplicaciones terapéuticas por existir aún experiencias limitadas.

Finalmente este estudio sirve de aporte teórico sobre la utilidad de LPD en los traumas abdominales penetrantes, los cuales requiere de mayor investigación y estudios exhaustivo, ya que se ha visto la controversia planteada, aun cuando es indudable el uso efectivo y eficiente del LPD en éstos, se precisa mayor especificidad en cuanto a las debilidades mencionadas en el diagnostico 
arrojado en estos casos.

\section{Bibliografía}

ATLAS. (2004). Programa Avanzado de Apoyo Vital en Trauma Para Médicos. Manual del curso $7^{\text {a }}$. México: Comité de Trauma de Colegio Americano de Cirujanos.

Burnwit, C., \& Thal, E. (1986). Significance of Omental Evisceration in Abdominal Stab Wounds. American Journal of Surgery, 152(6), 670-673. Retrieved from https://www.ncbi.nlm.nih.gov/pub$\mathrm{med} / 3789293$

Castillo Cruz, A. F. (2012, 10 23). Paracentesis y lavado peritoneal. Retrieved 01 2020, from https:// es.slideshare.net/AxelFerzho/paracentesis-y-lavado-peritoneal

Espinal, R., Ventura, O., \& Garcia, R. (1995). Traumatismo Abdominal. Revista Médica Hondureña, 63(4), 140-144. Retrieved from http://www.bvs.hn/ $\mathrm{RMH} / \mathrm{pdf} / 1995 /$ pdf/Vol63-4-1995-5.pdf

Feliciano, D. (1995). Modalidades de diagnóstico en el caso de traumatismo abdominal. Revista Médica Hondureña, 6(2), 243-248.

Ferrada, R., Garcia , A., Cantillo , E., Aristizábal, G., \& Abella, H. (2007). TRAUMA DE ABDOMEN. Bogota. Colombia: Asociacion Colombiana de Facultades de Medicina. Retrieved 01 20220, from http:// www.medynet.com/usuarios/jraguilar/Trauma\%20 Abdomen.pdf

Huizinga, W., Baker, L., \& Mtshali, Z. (1987). Selective management of abdominal and thoracic stab wounds with established peritoneal penetration. American Journal of Surgery, 153(6), 564-568. doi:10.1016/0002-9610(87)90155-3

Isaac L, J. P., Zapata Sirvent, R. L., Basso, I., Piñate , S., Marzullo, V., Blanco , A., . . Ibraín, G. (1989).
Lavado Peritoneal Diagnóstico en Trauma Abdominal Cerrado y Penetratante. Archivos del Hospital Vargas, 11-17.

Navas, C. A., \& González, J. (2004). Complicaciones neurológicas en cuidado intensivo. Asociación Colombiana de Neurología, 145-166.

Oreskovich, M. R., \& Carrico, C. J. (1983). Stab wounds of the anterior abdomen. Annals of surgery, 411-419.

Pacheco, A. (2011). Trauma de Abdomen. Revista Médica Clínica Las Condes, 623-630.

Pérez, N. (2018, 09 17). In Slide Share. Retrieved 01 2020, from https://es.slideshare.net/nadryperez/ trauma-abdominal-115081156

Presswalla, F. (1978, 10 18). PubMed. Retrieved 01 2020, from https://www.ncbi.nlm.nih.gov/pub$\mathrm{med} / 703563$

Root, H. D., HAuser, C. W., Mckinley, C. R., Lafave, J. W., \& Mendiola, R. J. (1965). Diagnostic peritoneal lavage. Surgery, 63 3- 637.

Saldarriaga L, M. (n.d.). Recursos Educacionales en español para la medicina de emergencia REEME. Retrieved 01 2020, from https://reeme.arizona. edu/directory.cfm?action=viewobjective\&objecti$\mathrm{ve}=651 \&$ objective_no $=38$

Ugarte Nuño, A., Etxeberria del Campo, A., Goienetxea Murgiondo, A., Biurrun Mancisidor, K., Esnaola Albizu, M., \& Fernández Martín, M. (2018, 11 15). Sociedad Española de Radiología Médica SERAM. Retrieved 01 2020, from https://piper.espacio-seram.com/index.php/seram/article/view/14

UNMSM. (1999). Cirugía: I cirugía general. Lima: Universidad Nacional Mayor de San Marcos (Lima). Facultad de Medicina. Escuela Académico Profesional de Medicina Humana. Departamento Académico de Cirugía.

\section{CITAR ESTE ARTICULO:}

Pazmiño Vargas, M., Saltos Morán, S., Coloma Pereira, J., \& Molina Villacres , M. (2020). Utilidad del lavado peritoneal en el trauma abdominal. RECIMUNDO, 4(1), 13-25. doi:10.26820/recimundo/4.(1).enero.2020.13-25 